\title{
TWIST1 upregulates miR-214 to promote epithelial-to-mesenchymal transition and metastasis in lung adenocarcinoma
}

\author{
CHAO LIU $^{1}$, JING LUO $^{1}$, YUE-TAO ZHAO ${ }^{1,2}$, ZHONG-YU WANG $^{1}$, JIE ZHOU $^{1}$, \\ SHUO HUANG ${ }^{1}$, JIA-NI HUANG ${ }^{1}$, HAI-XIA LONG ${ }^{1 *}$ and BO ZHU ${ }^{1 *}$ \\ ${ }^{1}$ Institute of Cancer, Xinqiao Hospital, Third Military Medical University, Chongqing 400037; \\ ${ }^{2}$ Department of Radiotherapy, 303 Hospital of PLA, Nanning 530021, P.R. China
}

Received December 23, 2016; Accepted February 20, 2018

DOI: $10.3892 /$ ijmm.2018.3630

\begin{abstract}
Epithelial-to-mesenchymal transition (EMT) is essential for the progression of non-invasive tumor cells into malignancy and metastasis. We found that miR-214 was increased in lung adenocarcinoma (LAD) and positively associated with metastasis, which was mediated by EMT. However, the mechanism whereby the overexpression of microRNAs (miRNAs), such as miR-214, promote EMT in LAD remains unclear. In this study, we found that TWIST1, an independent prognostic factor for overall survival, was increased in LAD and correlated positively with LAD recurrence and progression. We also found that TWIST1 contributes to the EMT process and metastasis of LAD cells. Most importantly, a positive correlation was found between the expression of miR-214 and TWIST1 in clinical LAD tissue. Additionally, miR-214 expression was decreased and its target gene suppressor of fused homolog $(S U F U)$ was increased in LAD cells in response to the impairment of TWIST1 expression by shRNA. Overall, this study provides the first evidence to show that the high expression of TWIST1 increases the expression of miR-214 to promote the EMT process and metastasis in LAD. These findings contribute to clarify the mechanisms whereby miRNAs regulate the EMT process and implicate a new TWIST1-miR-214 pathway in the control of migration and invasion of LAD.
\end{abstract}

Correspondence to: Dr Bo Zhu or Dr Hai-Xia Long, Institute of Cancer, Xinqiao Hospital, Third Military Medical University, Chongqing 400037, P.R. China

E-mail: b.davis.zhu@gmail.com

E-mail: longhaixialhx@163.com

${ }^{*}$ Contributed equally

Key words: lung adenocarcinoma, TWIST1, miR-214, metastasis epithelial-to-mesenchymal transition

\section{Introduction}

Tumor metastasis is one of the major causes of cancer-related deaths in patients with malignancies, so it is critical to understand the molecular mechanisms of effective therapeutic strategies to treat cancer. In recent years, a large and growing body of literature has investigated the involvement of the epithelial-mesenchymal transition (EMT) in the initiation of metastasis $(1,2)$. Through EMT, which is a developmental process, epithelial cells lose their adhesion properties and acquire mesenchymal features allowing them to migrate and invade $(3,4)$. In the tumor microenvironment, several molecules are involved in the EMT process. The transforming growth factor- $\beta$ (TGF- $\beta$ ) and hypoxia pathways are the best characterized and most frequently used signaling pathways in the EMT process (5-7). Transcription factors whose overexpression induces the EMT process in a variety of cancer cell lines include members of the Twist, Snail, Zeb and FoxC families (8).

MicroRNAs (miRNAs) are small, non-coding RNAs (containing $\sim 22$ nucleotides) that regulate gene expression post-transcriptionally by binding to complementary sequences within target mRNAs $(9,10)$. miRNAs have recently been identified as a class of factors that are involved in the EMT-MET switch during malignant tumor progression and metastasis. The miR-200 family of miRNAs cooperatively regulate the expression of the E-cadherin transcriptional repressors ZEB1/2 which is implicated in the EMT and tumor metastasis (11). Actually, our previous study revealed that the expression of miR-214 was elevated in lung adenocarcinoma (LAD) and correlated positively with LAD metastasis and EMT by targeting the suppressor of fused homolog (SUFU). Indeed this was the first evidence to demonstrate that the expression of miR-214 by LAD cells contributes to the EMT and metastasis in LAD (12). Additionally, miRNAs can also be regulated by some molecules. For instance, the tumor suppressor $p 53$ targets miR-34a and miR-215 to repress SNAIL and ZEB2 expression (13). However, the mechanisms upstream of the miRNAs remain largely unknown.

TWIST1, a basic-helix-loop-helix (bHLH) transcription factor, is one of the most important EMT-inducer prototypes (14). TWIST1 binds to the E-box elements of E-cadherin 
Table I. Primers for selected genes.

Primers

Gene

name

Sense

Antisense

Product (bp)

E-cadherin

Vimentin

SUFU

TWIST1

$\beta$-actin
GTCTGTCATGGAAGGTGCT

CCACGAAGAGGAAATCCAGG

GCCTGAGTGATCTCTATGGTGA

GTCCGCAGTCTTACGAGGAG

GAGCTACGAGCTGCCTGACG
TACGACGTTAGCCTCGTTC

320

CAGAGAGGTCAGCAAACTTGG

TCTCTCTTCAGACGAAAGGTCAA

GCTTGAGGGTCTGAATCTTGCT

GTAGTTTCGTGGATGCCACAG

\section{8}

100

156

120

SUFU, suppressor of fused homolog.

and represses its expression, whereas it promotes $\mathrm{N}$-cadherin expression by binding to the E-box elements of N-cadherin (15). In addition, TWIST1 regulates the expression of miRNAs. For instance, TWIST1 upregulates miR-10b to promote breast cancer metastasis (16). miR-223, which is directly induced by TWIST1, could promote migration and invasion in gastric cancer cells (17). More importantly, TWIST1 have been shown to drive the expression of a 7.9-kb noncoding RNA transcript that encodes a miR-199a and miR-214 cluster (18). As expressed above, we hold the hypothesis that the miR-214 expression is elevated by TWIST1 during the EMT process in LAD cells.

In this study, our data demonstrated that TWIST1 was highly expressed by EMT and high expression of TWIST1 upregulates miR-214 to promote the EMT process and metastasis in LAD.

\section{Materials and methods}

Cell culture and patient samples. The human LAD cell lines A549 and NCI-H1650 were obtained from the American Type Culture Collection (ATCC, Manassas, VA, USA). These cell lines were last tested and authenticated by short tandem repeat profiling in September, 2014. The cell lines were maintained in Dulbecco's modified Eagle's medium (DMEM) with $10 \%$ fetal bovine serum (FBS), $100 \mathrm{U} / \mathrm{ml}$ penicillin, and $100 \mu \mathrm{g} / \mathrm{ml}$ streptomycin, and were incubated at $5 \% \mathrm{CO}_{2}$ at $37^{\circ} \mathrm{C}$. In experiments designed to induce EMT by hypoxia, the cells were cultured under normoxic conditions $\left(21 \% \mathrm{O}_{2}\right)$ or hypoxic conditions $\left(0.5 \% \mathrm{O}_{2}\right)$ for $24 \mathrm{~h}$, as previously detailed in the literature $(6,7)$. To induce the EMT with TGF- $\beta$ or paclitaxel, cells were treated with TGF- $\beta(5 \mathrm{ng} / \mathrm{ml})$ for 7 days (6) or with paclitaxel $(5 \mathrm{ng} / \mathrm{ml})$ for 2 days (19), and then the cells were collected for further study. LAD tissue samples and their corresponding paracancerous tissue samples were collected by surgical resection at Daping Hospital (Chongqing, China) from August, 2011 to September, 2013. Fresh primary and metastatic LAD tissue samples were collected at Xinqiao Hospital (Chongqing, China) from August, 2012 to September, 2014. This study was approved by the Institutional Review Board of the Third Military Medical University, and informed consent was obtained from each patient.

RNA extraction and real-time PCR. miRNA expression levels were measured using the qRT-PCR miRNA kit and
qRT-PCR primer sets, according to the manufacturer's instructions (Ribobio, Guangzhou, China). Primers for the human E-cadherin, vimentin, SUFU, TWIST1 and $\beta$-actin $(A C T B)$ are listed in Table I. Real-time PCR was performed in an ABI 7500 Prism Sequence Detection system (Applied Biosystems, Foster City, CA, USA) using a SYBR-Green kit (Takara, Tokyo, Japan), and the relative changes in expression were quantified. Each experiment was repeated at least three times.

RNA interference. LAD cells were stably infected with the premicroRNA expression construct known as the lenti-miR expression plasmid, which contained the full-length miR-214 in the H1-MCS-CMV-EGFP vector (GeneChem Inc., Shanghai, China; vector information: http://www.genechem.com.cn/Zaiti. aspx?zt=GV259). The sh-miR-214 sequence (ACTGCCTG TCTGTGCCTGCTGT) was cloned into the H1-MCS-CMVEGFP (vector information: http://www.genechem.com.cn/Zaiti. aspx?zt=GV159) to generate the H1-MCS-CMV-EGFPsh-miR-214 (both from GeneChem Inc.). For the knockdown of TWIST1, TWIST1-specific shRNAs (shRNA1, GCUGAGC AAGAUUCAGACCTT; shRNA2, GGUCUGAAUCUUGCU CAGCTT) were packaged into the lentivirus from GeneChem Inc. A non-targeting sequence, purchased from GeneChem Inc. was used as a lentivirus negative control. An infection efficiency $>80 \%$ was verified by fluorescent microscopy and confirmed for TWIST1 and miR-214 expression.

Migration and invasion assay. The migration assay was performed using 24-well culture inserts with a porous (pore size $8.0 \mu \mathrm{m}$ ) polycarbonate membrane (Millipore, Billerica, MA, USA). For the Matrigel invasion assay, the filters were precoated with $30 \mu 1$ of Matrigel (BD Biosciences, Franklin Lakes, $\mathrm{NJ}$, USA) for $3 \mathrm{~h}$. The migration and invasion assays were performed following our previously published protocol (20). Briefly, $2 \times 10^{4}$ cells in $200 \mu \mathrm{l}$ of serum-free medium were added to the upper chamber, and $800 \mu$ l of medium with $5 \%$ serum was added to the lower chamber. The plates were incubated for $24 \mathrm{~h}$ at $37^{\circ} \mathrm{C}$ in $5 \% \mathrm{CO}_{2}$. Cells that did not migrate or invade through the pores were removed with a cotton swab. Cells on the lower surface of the membrane were examined and counted under a microscope. Each experiment was repeated at least three times.

Flow cytometry sorting. Dissociated LAD cells, which were stably infected with the pre-microRNA expression, 

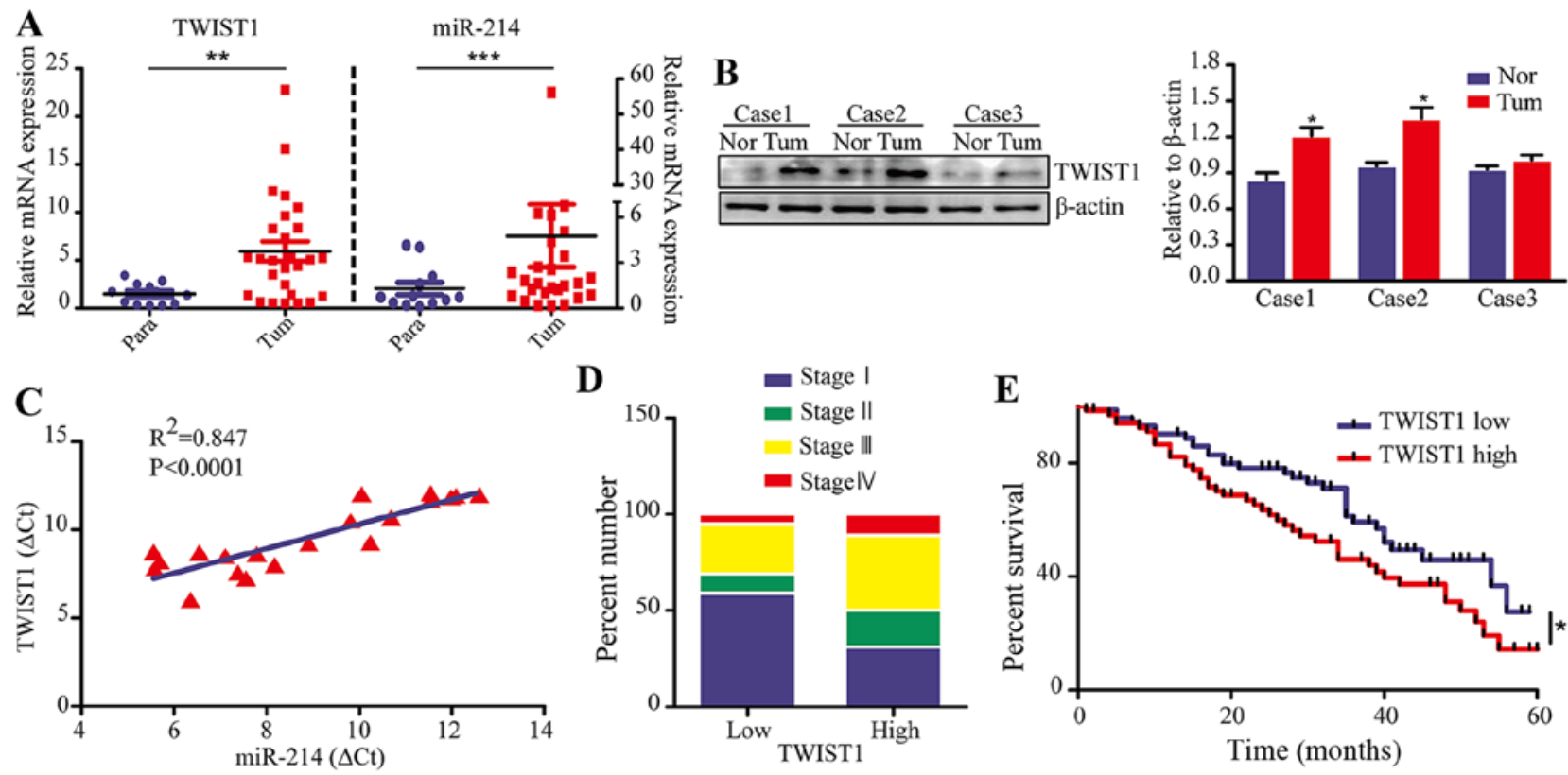

Figure 1. TWIST1 is a negative prognostic indicator in lung adenocarcinoma (LAD) patients and positively correlates with miR-214 expression. (A) The quantitation of TWIST1 or miR-214 expression in human primary LAD cancer tissues $(n=22)$ and paracancerous tissues $(n=13)$. The relative expression levels were normalized to the mean value of all patients. (B) Western blot analyses of TWIST1 expression in 3 cases of LAD tissue. (C) The positive correlation between miR-214 and TWIST1 expression in 20 clinical samples was determined by Pearson's correlation coefficient $\left(\mathrm{R}^{2}=0.847, \mathrm{P}<0.0001\right)$. (D) The correlation between the TWIST1 levels and clinical pathological parameters in LAD patients. (E) Kaplan-Meier curves of survival differences between LAD patients. Percent survival for patients expressing high and low levels of TWIST1. P-values were determined by the log-rank test. All experiments were performed at least three times. The statistical significance of differences was measured by unpaired Student's t-test. ${ }^{*} \mathrm{P}<0.05,{ }^{* *} \mathrm{P}<0.01$ and ${ }^{* * * *} \mathrm{P}<0.001$.

Table II. Association between TWIST1 expression in LAD patients' characteristics.

\begin{tabular}{lrrrr}
\hline & \multicolumn{4}{c}{ TWIST1 } \\
\cline { 2 - 5 } & Total & High & Low & P-value \\
\hline Age & & & & \\
$\geq 60$ & 84 & 45 & 39 & \\
$<60$ & 16 & 5 & 11 & 0.101 \\
Sex & & & & \\
$\quad$ Male & 50 & 30 & 22 & \\
Female & 48 & 20 & 28 & 0.109 \\
Tobacco history & & & & \\
$\quad$ Yes & 83 & 44 & 39 & \\
No & 17 & 6 & 11 & 0.183 \\
Vital statistics & & & & \\
Alive & 43 & 27 & 16 & \\
Death & 57 & 23 & 34 & 0.026 \\
Recurrence & & & & \\
$\quad$ Yes & 36 & 23 & 13 & \\
No & 64 & 27 & 37 & $0.037^{\mathrm{a}}$ \\
Final stage & & & & \\
I & 53 & 19 & 34 & \\
II & 18 & 13 & 5 & \\
III & 27 & 18 & 9 & $0.005^{\mathrm{b}}$ \\
IV & 2 & 2 & 0 & \\
\hline
\end{tabular}

The statistical significance of difference was measured by Pearson's $\chi^{2}$ test. ${ }^{\mathrm{a}} \mathrm{P}<0.05$ and ${ }^{\mathrm{b}} \mathrm{P}<0.01$. were counted and transferred to a 5-ml tube, washed twice with phosphate-buffered saline (PBS), counted and resuspended in PBS at $1 \times 10^{6}$ cell $/ 100 \mu$ l. The samples were then suspended in $500 \mu 1$ of PBS. The cells were routinely sorted twice and they were analyzed for GFP purity, which was typically $>80 \%$. The data were analyzed with the CellQuest software (BD Biosciences).

Immunofluorescence. The immunofluorescence analysis was performed on 8- $\mu \mathrm{m}$-thick frozen sections of tissue, which were fixed with ice-cold $4 \%$ paraformaldehyde for $15 \mathrm{~min}$ and blocked with normal serum for $20 \mathrm{~min}$ at room temperature before being incubated with one or more specific antibodies against vimentin (1:200), E-cadherin (1:200), SUFU (1:200) or TWIST1 (1:200) (all from Abcam, Cambridge, UK) overnight and in the dark at $4^{\circ} \mathrm{C}$. After three washes, the slides were stained with FITC-conjugated anti-rabbit antibodies or Cy3-conjugated anti-mouse antibodies (1:500; Abcam). The nuclei were counterstained with 4',6-diamidino-2-phenylindole (DAPI). Stained cells were visualized with an Olympus confocal microscope (Olympus Corp., Tokyo, Japan). All the experiments were repeated at least three times.

Western blot analysis. All the cell lysates were prepared and western blot analysis was performed as previously described (20). The following antibodies were used: E-cadherin (1:500), vimentin (1:500) (both from Abcam), SUFU (1:200; Santa Cruz Biotechnology, Inc., Santa Cruz, CA, USA), TWIST1 (1:200; BD Biosciences), and $\beta$-actin (1:400; Boster, Wuhan, China).

Statistical analyses. The data are presented as the means \pm SD. The data were statistically analyzed by the Student's t-test or a 

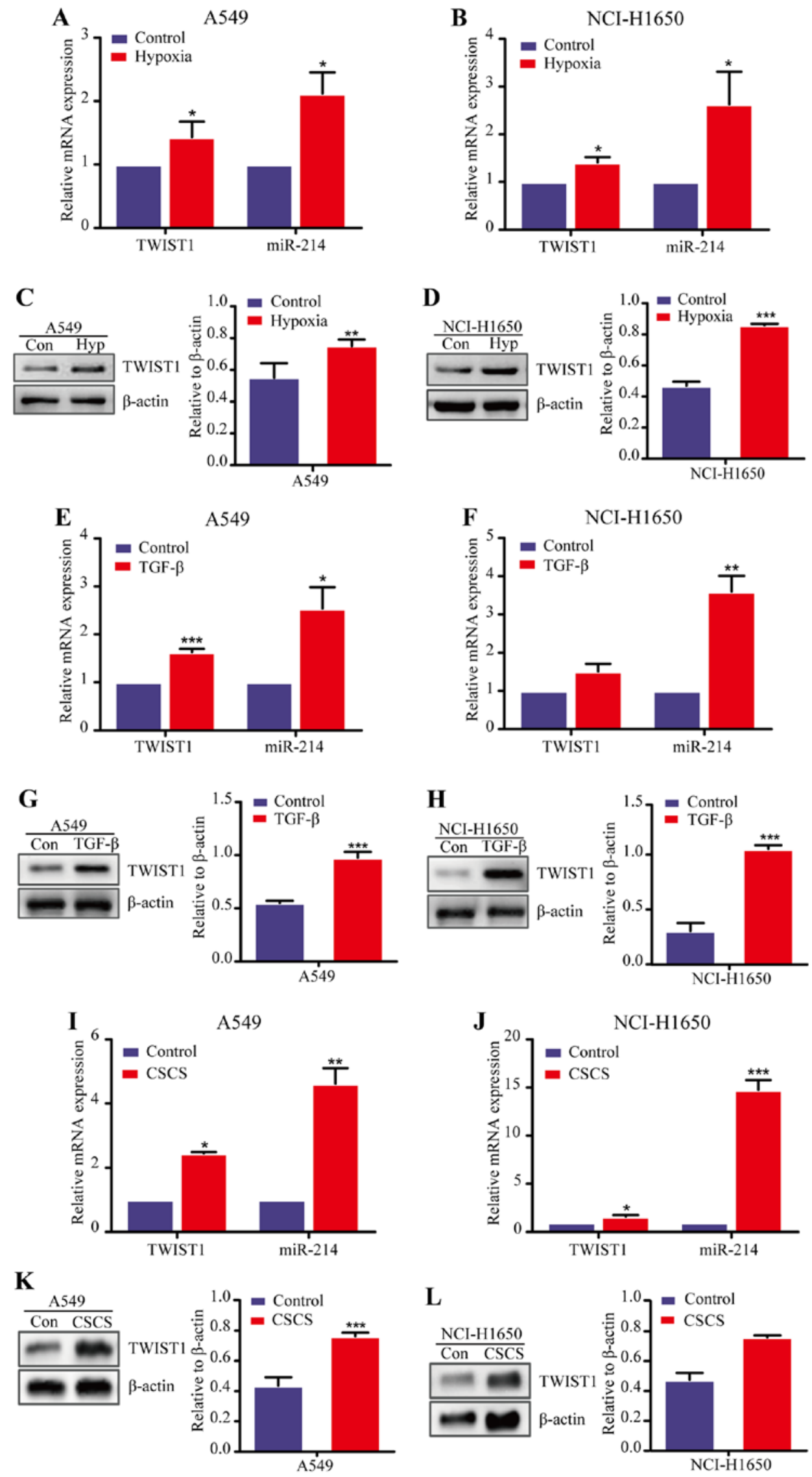

Figure 2. TWIST1 was increased in the epithelial-to-mesenchymal transition (EMT) of lung adenocarcinoma (LAD) cells. (A and B) Real-time PCR analyses of TWIST1 and miR-214 expression in two LAD cells (A549 and NCI-H1650) under normoxia $\left(21 \% \mathrm{O}_{2}\right)$ or hypoxia $\left(0.5 \% \mathrm{O}_{2}\right)$ treatment for $24 \mathrm{~h}$. (C and D) Western blot analyses of TWIST1 expression in A549 (C) and NCI-H1650 cells (D) under normoxia $\left(21 \% \mathrm{O}_{2}\right)$ or hypoxia $\left(0.5 \% \mathrm{O}_{2}\right)$ treatment for $24 \mathrm{~h}$. (E-H) TWIST1 and miR-214 expression in two LAD cells (A549 and NCI-H1650) under transforming growth factor- $\beta$ (TGF- $\beta$ ) (5 ng/ml) treatment for 7 days. (I-L) TWIST1 and miR-214 expression in CSCs isolated from two LAD cells (A549 and NCI-H1650). All experiments were performed at least three times and the data are expressed as the means $\pm \mathrm{SD}$. The statistical significance of differences was measured by unpaired Student's t-test. ${ }^{*} \mathrm{P}<0.05,{ }^{* *} \mathrm{P}<0.01$ and ${ }^{* * *} \mathrm{P}<0.001$. 
A

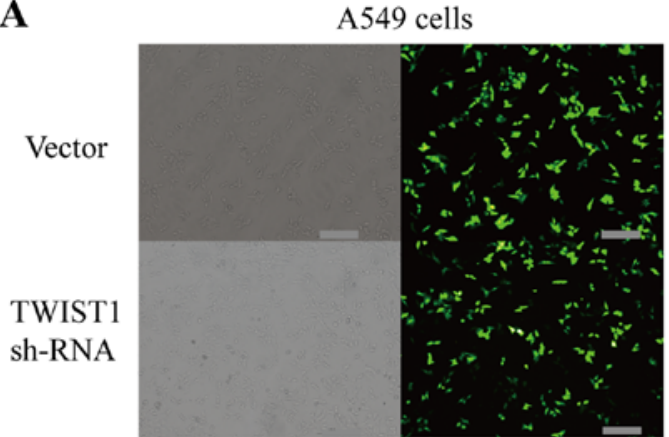

NCI-H1650 cells

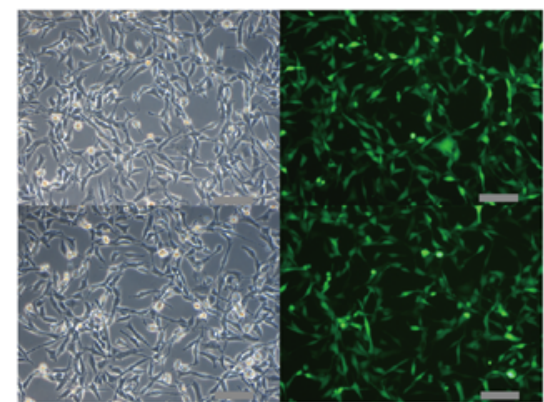

B

Vector
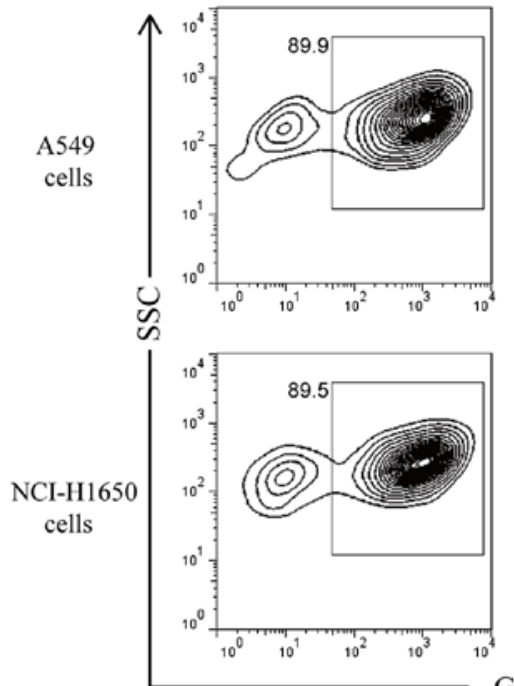

TWIST1 sh-RNA
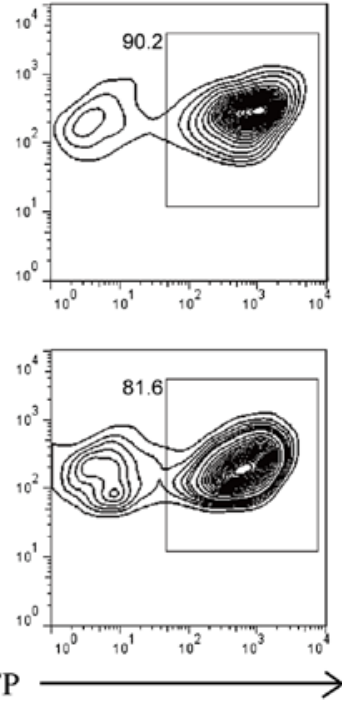

C

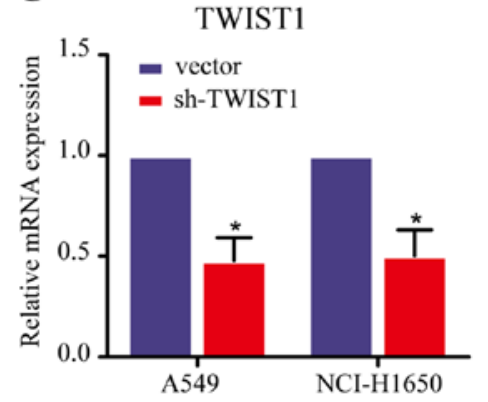

Figure 3. Stable TWIST1 downregulation cell lines were established by using lentivirus transfection. (A) A morphological image of A549 and NCI-H1650 cells transfected with TWIST1 inhibitor or empty lentiviral vectors. (B) The flow sorting of A549 and NCI-H1650 cells transfected with TWIST1 inhibitor or empty lentiviral vectors. (C) TWIST1 expression levels as measured by qRT-PCR in the indicated cells following transfection with the above-mentioned virus. All experiments were performed at least three times and the data are expressed as the means \pm SD. The statistical significance of differences was measured by unpaired Student's t-test. ${ }^{*} \mathrm{P}<0.05$.

one-way ANOVA test. A difference was considered to be statistically significant at $\mathrm{P}<0.05$. All the statistical analyses were performed with the SPSS 13.0 software (IBM Corp., Armonk, NY, USA).

\section{Results}

TWIST1 is a poor prognostic indicator in LAD and positively correlates with miR-214 expression. We assessed the expression of TWIST1 and miR-214 in 22 primary and 13 paracancerous LAD tissue samples by quantitative real-time PCR (qRT-PCR) analysis. The results revealed that the TWIST1 expression was significantly higher in tumor tissues compared with paracancerous tissues $(\mathrm{P}<0.01)$, which is the same trend as observed with the expression of miR-214 (Fig. 1A). Additionally, western blot analysis showed that TWIST1 expression was higher in tumor tissues compared to non-tumor tissue (Fig. 1B). More importantly, we found a positive correlation between miR-214 expression and TWIST1 expression in 20 cases of clinical LAD tissue $\left(\mathrm{P}<0.0001, \mathrm{R}^{2}=0.847\right)$ (Fig. 1C). To investigate the potential roles of TWIST1 in LAD, we analyzed the correlation between the TWIST1 levels and the clinical pathological parameters in LAD patients (gene expression data were obtained from the NCBI GEO datasets, GPL3877). The results revealed that patients with the most advanced stage cancer (stage III and IV) had high TWIST1 expression (>50\%). Conversely, patients with early stage cancer (stage I) had low TWIST1 expression (>50\%) (Fig. 1D). In addition, the expression of TWIST1 was significantly correlated with recurrence and final stage of LAD patients (Table II). However, there was no significant correlation between TWIST1 expression and age, sex, tobacco history or vital statistics (all $\mathrm{P}>0.05$ ). Comparison of the survival curves of LAD patients revealed that TWIST1-positive (high expression) patients had significantly poorer survival than TWIST1-negative (low expression) patients $(\mathrm{P}<0.05)$ (Fig. 1E). Further evaluation of these findings by univariate analysis revealed that the TWIST1 status was an independent prognostic factor for survival. In fact, TWIST1-positive patients were more likely to suffer from relapse than TWIST1-negative patients [hazard ratio (HR), 2.057; 95\% confidence interval (95\% CI), 1.030-4.111]. On the other hand, no association was found between the patient's prognosis and sex, age or tobacco history. Additionally, multivariate Cox regression analyses confirmed that higher expression of TWIST1 was indeed an independent prognostic factor of survival of LAD patients (HR, 2.201; 95\% CI, 1.192-4.062), whereas sex, age or tobacco history were not independent prognostic factors (Table III). 
Table III. Univariate and multivariate analysis of variables with overall survival.

\begin{tabular}{|c|c|c|c|c|}
\hline \multirow[b]{2}{*}{ Variables } & \multicolumn{2}{|c|}{ Univariate } & \multicolumn{2}{|c|}{ Multivariate } \\
\hline & HR $(95 \% \mathrm{CI})$ & P-value & $\mathrm{HR}(95 \% \mathrm{CI})$ & P-value \\
\hline Sex (female vs. male) & $1.480(0.773-2.759)$ & 0.243 & & \\
\hline Age $(\geq 60$ vs. $<60)$ & $0.516(0.233-1.145)$ & 0.104 & & \\
\hline Tobacco history (no vs. yes) & $1.316(0.487-3.553)$ & 0.588 & & \\
\hline TWIST1 (low vs. high ) & $2.057(1.030-4.111)$ & $0.041^{\mathrm{a}}$ & $2.201(1.192-4.062)$ & $0.012^{\mathrm{a}}$ \\
\hline
\end{tabular}

Cox proportional hazards regression model. ${ }^{\mathrm{a}} \mathrm{P}<0.05$. HR, hazard ratio; $\mathrm{CI}$, confidence interval.
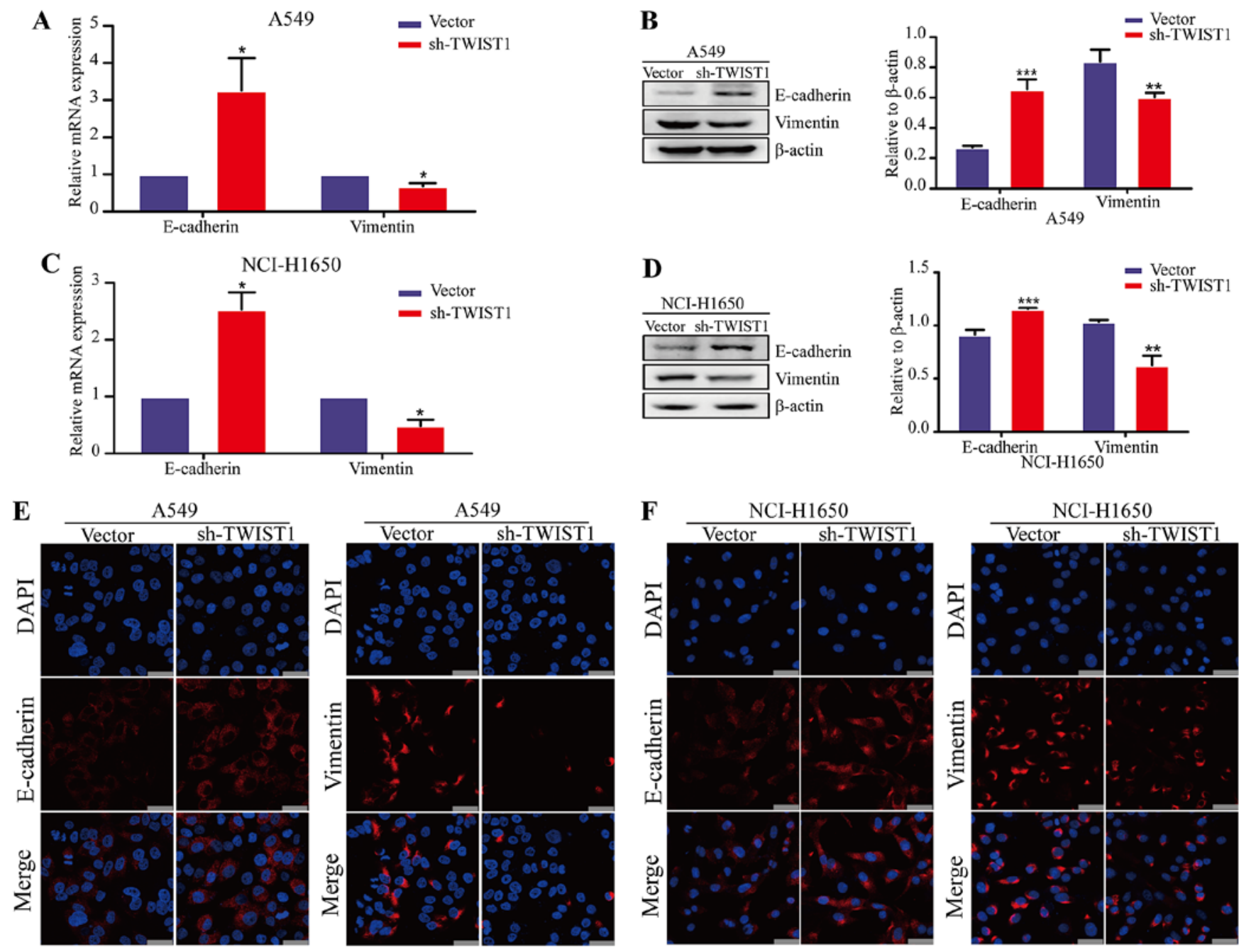

Figure 4. Downregulation of TWIST1 suppresses the epithelial-to-mesenchymal transition (EMT) process in lung adenocarcinoma (LAD) cells. (A and B) The expression of EMT markers analyzed in sh-TWIST1-transfected in A549 cells by real-time PCR (A) or western blotting (B). (C and D) The expression of EMT markers analyzed in sh-TWIST1-transfected in NCI-H1650 cells by real-time PCR (C) or western blotting (D). (E and F) The expression of EMT markers was analyzed by immunofluorescent staining in sh-TWIST1-transfected A549 cells (E) and NCI-H1650 (F). The nuclei were stained with DAPI. Scale bar, $25 \mu \mathrm{m}$. All experiments were performed at least three times and the data are expressed as the means \pm SD. The statistical significance of differences was measured by unpaired Student's t-test. ${ }^{*} \mathrm{P}<0.05,{ }^{* *} \mathrm{P}<0.01$ and ${ }^{* * *} \mathrm{P}<0.001$.

Collectively, our findings indicate a positive correlation between the expression of miR-214 and TWIST1, and TWIST1 was an important prognostic factor of LAD patients.

TWIST1 expression is increased in the EMT of LAD cells. Our previous studies revealed the important role played by miR-214 in the EMT process and metastasis of LAD cells, as well as the positive correlation between the expression of miR-214 and TWIST1. Accordingly, we further investigated whether TWIST1 promoted LAD metastasis through the EMT process. Previous studies described that the role of TGF- $\beta$ and hypoxia in the EMT process were identified as the best characterized 

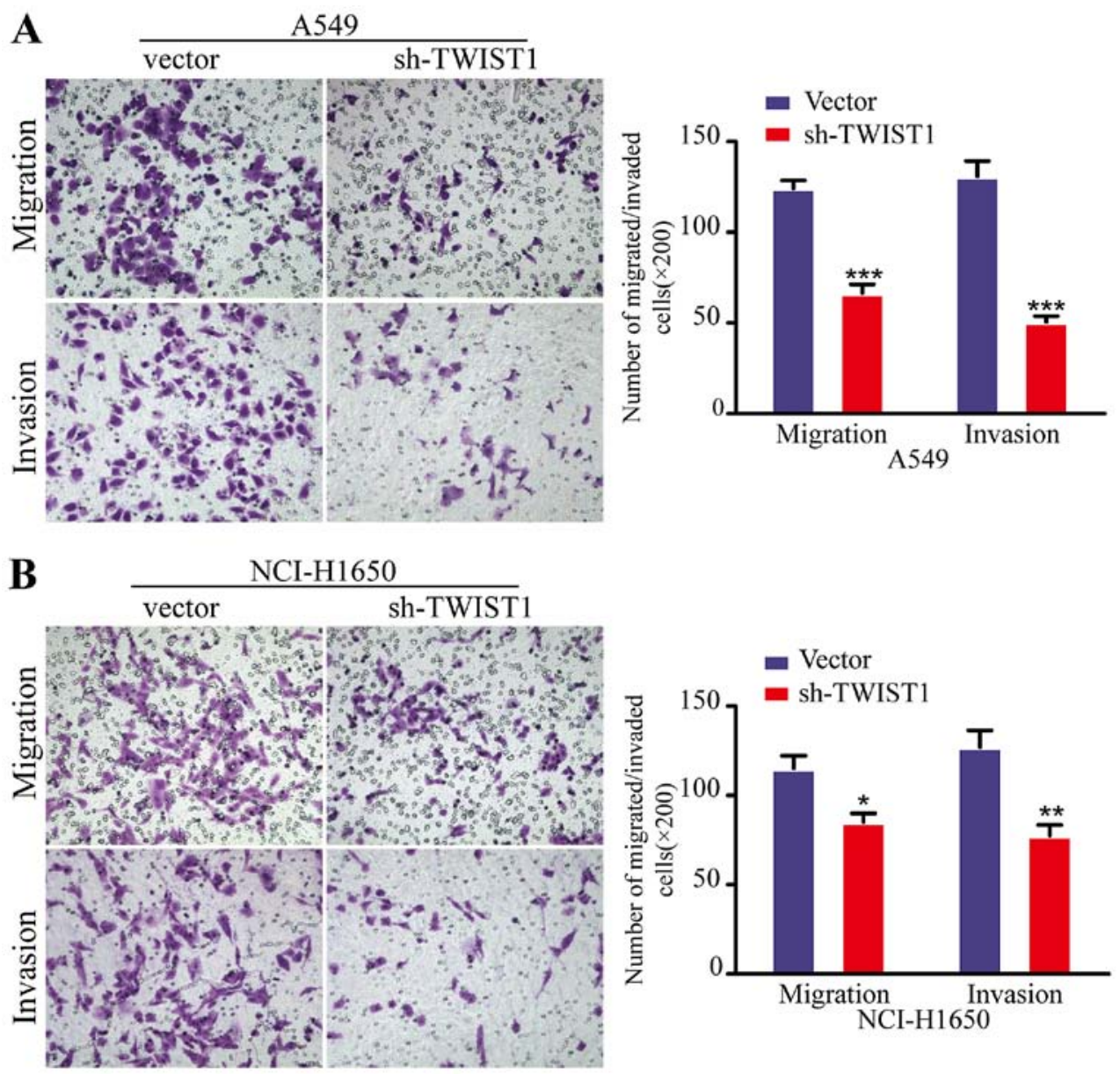

Figure 5. Sh-TWIST1 impairs lung adenocarcinoma (LAD) cell migration and invasion in vitro. (A and B) The migration and invasion abilities of A549 (A) and NCI-H1650 cells (B) when transfected with the sh-TWIST1-transfected vector or an empty vector as assessed by Transwell migration and Matrigel invasion assay for $24 \mathrm{~h}$. All experiments were performed at least three times. The statistical significance of differences was measured by unpaired Student's $\mathrm{t}$-test. ${ }^{*} \mathrm{P}<0.05,{ }^{* *} \mathrm{P}<0.01$ and ${ }^{* * *} \mathrm{P}<0.001$.

inducers of EMT, and some researchers indicated that cancer stem cells (CSCs) displayed an epithelial-mesenchymal transition phenotype (21). Thus, we first established a hypoxiainduced EMT model using two LAD cell lines (A549 and NCI-H1650) and used qRT-PCR analysis to measure the expression of TWIST1 and miR-214. We found that both the TWISTI and miR-214 were upregulated in the two LAD cell lines after hypoxic $\left(0.5 \% \mathrm{O}_{2}\right)$ induction for $24 \mathrm{~h}$ compared with normoxia condition $\left(21 \% \mathrm{O}_{2}\right)$ (Fig. $2 \mathrm{~A}$ and B). In addition, western blot analysis confirmed that TWIST1 expression was significantly increased at the protein level in A549 and NCI-H1650 cells after exposure to hypoxia (Fig. 2C and D). Similar results were also consistently obtained in the TGF- $\beta$-induced EMT model and CSCs $(12,22)$ (Fig. 2E-L). Together, these data demonstrate that the TWIST1 expression was increased in the EMT cells of LAD in parallel with an increased expression of miR-214, suggesting that TWIST1 may activate the EMT process in LAD in association with the elevated expression of miR-214.

Sh-TWIST1 impaires the EMT process in LAD cells. As described above, we found that TWIST1 expression was increased in the EMT of LAD cells. Considering these findings, we focused on TWIST1 for further pro-EMT functional studies. Accordingly, we employed a loss-of-function approach by using shRNA in A549 and NCI-H1650 cells to investigate the role of TWIST1 in the EMT process. We first generated LAD cell lines with stable TWIST1 downregulation (A549 and NCI-H1650) using lentivirus transfection (Fig. 3). Fluorescent microscopy (Fig. 3A), flow cytometry (Fig. 3B) and qRT-PCR (Fig. 3C) analyses demonstrated that, as intended, the expression of TWIST1 was successfully downregulated in the NCI-H1650 and A549 cells. Indeed, the epithelial marker E-cadherin was significantly increased, whereas the mesenchymal marker vimentin was significantly decreased at both the gene and protein levels after the loss of expression of TWIST1 in LAD cells (Fig. 4A-D). Moreover, similar results were confirmed in LAD cell lines by an immunofluorescence approach (Fig. 4E and F). Taken together, these data indicate that sh-TWIST1 could weaken the EMT process in LAD cells.

Sh-TWIST1 impairs LAD cell migration and invasion in vitro. The EMT process is one of the key initiation steps in metastatic progression, which provides cancer cells with motility, invasion and migration properties. Accordingly, we next employed the Boyden chamber migration/invasion technique to investigate the function of TWIST1 in metastasis of LAD cells in vitro. As anticipated, the downregulation of TWIST1 by shRNA dramatically decreased the migratory and invasive abilities of both the A549 and NCI-H1650 cells (Fig. 5), compared with 
A

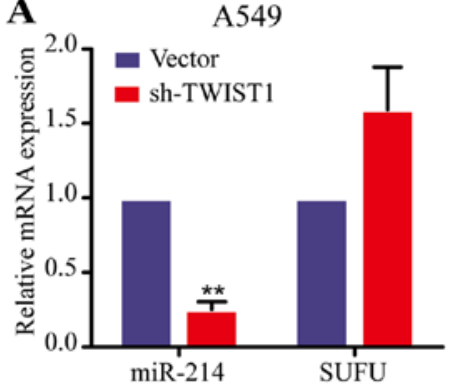

C

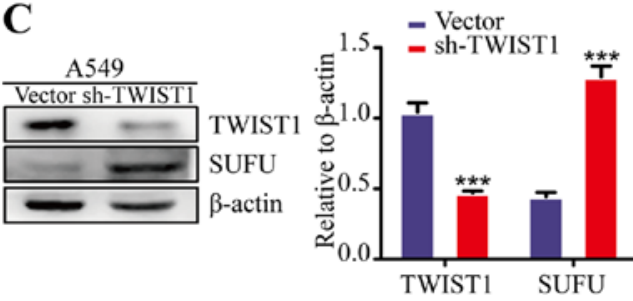

$\mathbf{E}$
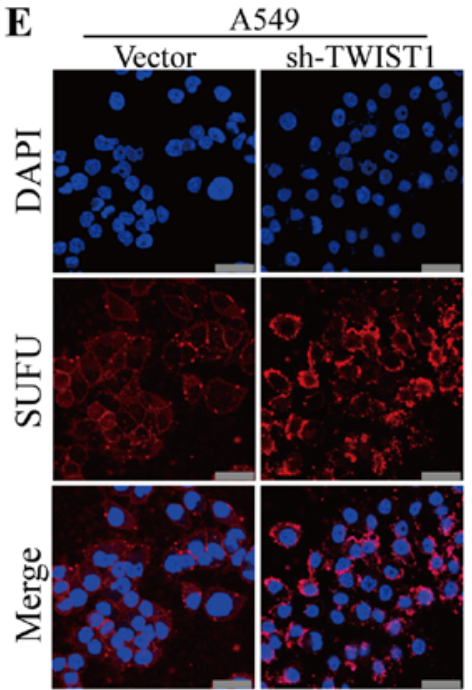

B

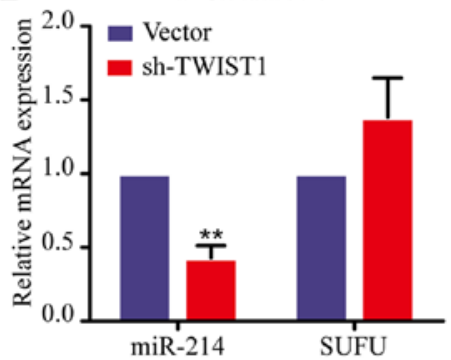

D
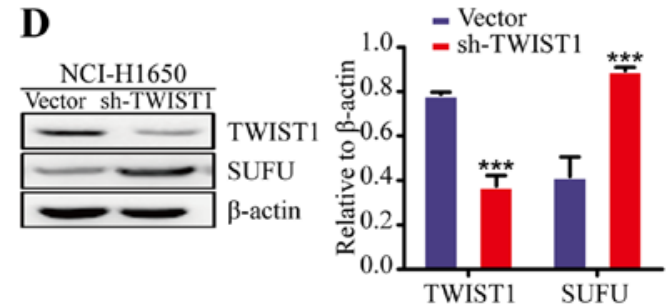

F

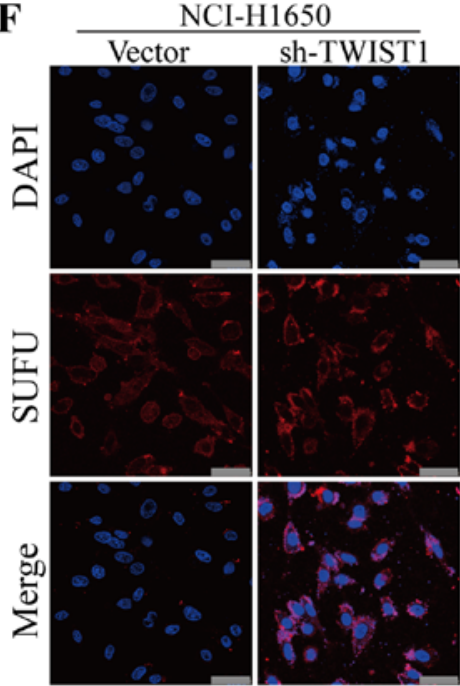

Figure 6. TWIST1 upregulates miR-214 in lung adenocarcinoma (LAD). (A and B) The expression of miR-214 and suppressor of fused homolog (SUFU) analyzed in sh-TWIST1-transfected A549 (A) or NCI-H1650 cells (B) by real-time PCR. (C and D) The expression of SUFU analyzed in sh-TWIST1-transfected A549 (C) or NCI-H1650 cells (D) by western blotting. (E and F) The expression of SUFU analyzed in sh-TWIST1-transfected A549 (E) or NCI-H1650 cells (F) by immunofluorescence. The nuclei were stained with DAPI. Scale bar, $25 \mu \mathrm{m}$. All experiments were performed at least three times and the data are expressed as the means $\pm \mathrm{SD}$. The statistical significance of differences was measured by unpaired Student's t-test. ${ }^{*} \mathrm{P}<0.05,{ }^{* * *} \mathrm{P}<0.01$ and ${ }^{* * * *} \mathrm{P}<0.001$.

cells transfected with the control vector. Collectively, these data suggested that sh-TWIST1 significantly moderated LAD cell metastasis in vitro.

TWIST1 upregulates miR-214 expression in LAD. Previous research revealed that TWIST1 drives the expression of a 7.9-kb noncoding RNA transcript that encodes the miR199a and miR-214 cluster (18). Thus, we hypothesized that miR-214 may be upregulated by TWIST1 in LAD cells. To prove this hypothesis we analyze the effects of TWIST1 on miR-214 expression. The results of the qRT-PCR analysis showed that the miR-214 expression was decreased in TWIST1 knockdown LAD cells, compared with the control vector group (Fig. 6A and B). Our previous studies identified $S U F U$ as a direct target of miR-214 in LAD cells (12). To determine whether TWIST1 inhibition, mimicking miR-214 expression, is sufficient to regulate $S U F U$ expression, we examined the SUFU expression in TWIST1 knockdown LAD cells. TWIST1 knockdown increased SUFU levels in the two LAD cell lines (A549 and NCI-H1650) by qRT-PCR, western blot and immunofluorescence analyses (Fig. 6C-F). These data are consistent with our hypothesis that TWIST1 induces miR-214 to promote the EMT process and metastatic progression in LAD.

\section{Discussion}

LAD is the most common form of lung cancer. Approximately $90 \%$ of LAD patients develop distant metastasis at the advanced stage (23). A better understanding of the molecular mechanisms underlying distant metastasis is necessary to facilitate the development of effective therapeutic strategies for LAD patients. While TWIST1 is known for its ability to induce EMT and tumor progression (24-26), to our knowledge, little is known about TWIST1 being a negative prognostic factor in LAD. In this study, TWIST1 was found to be increased and correlated with recurrence and final stage in LAD patients. Moreover, LAD patients with high expression of TWIST1 
have a poor survival rate. Univariate and multivariate analyses revealed that TWIST1 was an independent prognostic factor for overall survival of patients with LAD. Thus, our findings establish a previously unrecognized and important role for TWIST1 in the recurrence of different stages of LAD patients and malignant progression.

TWIST1 functions as a negative regulator of epithelial gene expression and a positive regulator of mesenchymal gene expression, leading to induction of the EMT $(15,27)$. In this study, TWIST1 was found to be upregulated in two LAD cell lines after hypoxic induction. Additionally, similar results were also consistently obtained in the TGF- $\beta$-induced EMT model and CSCs. Moreover, downregulation of TWIST1 in LAD cells upregulated E-cadherin, downregulated vimentin and impaired LAD cells migration and invasion. These data indicate that TWIST1 contribute to the EMT process and metastasis of LAD cells.

Prior studies have noted that TWIST1 regulates the expression of several miRNAs, which leads to induction of EMT. Here, for the first time, we report a positive correlation between miR-214 expression and TWIST1 expression in clinical LAD tissue samples. We also demonstrated that knockdown of TWIST1 expression in LAD not only represses the expression of miR-214, but also leads to an increased expression of the target gene $S U F U$ in two different cell lines. These data indicate that TWIST1 levels clearly upregulate miR-214 expression and as a result enhance the induction of EMT and metastasis in LAD. Nevertheless, Li et al (28) reported that in human intrahepatic cholangiocarcinoma cells miR-214 represses TWIST1 expression by targeting the 3'UTR of TWIST1, and downregulation of miR-214 promotes the EMT by directly targeting the TWIST1. In this study, we performed experiments in two different LAD cell lines and provide strong evidence with clinical samples. Moreover, we also demonstrated that miR-214 enhanced the EMT of LAD cells, an important process involved in metastatic progression, supporting a promoting function of miR-214 in LAD metastasis. However, it may be explained by the following reasons: i) both findings indicate an intricate interaction network between miRNAs and TWIST1, which is involved in different stages of cancer progression through regulating the EMT process. Thus, while the high level of TWIST1 upregulates miR-214 expression, miR-214 may further impair TWIST1 as a feedback mechanism. ii) The contrasting results may be related to the use of different cell lines, since the same miRNA could perform different functions through distinct pathways in a way that was dependent on the tissue or cell type (29). iii) A single gene has complex functions, it can play a dual role in distinct mechanisms to regulate tumor growth depending on the specific situation. However, this is no way to fully define the function of one gene. For instance, a review of the literature reveals that TWIST1 overexpression in mammary epithelial and cancer cell lines has been shown to promote tumor stemness (25), and miR-214 enhances the stemness and self-renewal of cancer stem-like cells (CSLCs) in lung adenocarcinomas by targeting CTNNBIP1 (22). This indicates that TWIST1 may play a critical role in CSLCs self-renewal and stemness by upregulating miR-214 expression.

Collectively, this study has significant implications for understanding the underlying mechanisms of how TWIST1 elevates the expression of miR-214 to contribute EMT, tumor metastasis and poor clinical outcomes in LAD. The knowledge on crosstalk between miR-214 and TWIST1 provides new potential diagnostic and therapeutic strategies in LAD treatment.

\section{Acknowledgements}

Not applicable.

\section{Funding}

This study was supported by the National Nature Science Foundation of China (no. 81773041).

\section{Availability of data and material}

The datasets used and/or analyzed during the current study are available from the corresponding author on reasonable request.

\section{Authors' contributions}

CL, HXL and BZ conceived and designed the study. CL, JL, YTZ, ZYW, JZ, SH and JNH performed the experiments. CL wrote the paper. CL, HXL and BZ reviewed and edited the manuscript. All authors read and approved the manuscript.

\section{Ethics approval and consent to participate}

This study was approved by the Institutional Review Board of the Third Military Medical University, and informed consent was obtained from each patient.

\section{Consent for publication}

Not applicable.

\section{Competing interests}

The authors declare that they have no competing interests.

\section{References}

1. Thiery JP and Lim CT: Tumor dissemination: An EMT affair. Cancer Cell 23: 272-273, 2013.

2. Brabletz T: EMT and MET in metastasis: Where are the cancer stem cells? Cancer Cell 22: 699-701, 2012.

3. Kalluri R and Weinberg RA: The basics of epithelial-mesenchymal transition. J Clin Invest 119: 1420-1428, 2009.

4. Thiery JP, Acloque H, Huang RY and Nieto MA: Epithelialmesenchymal transitions in development and disease. Cell 139: 871-890, 2009.

5. Gunaratne A, Thai BL and Di Guglielmo GM: Atypical protein kinase $\mathrm{C}$ phosphorylates Par6 and facilitates transforming growth factor $\beta$-induced epithelial-to-mesenchymal transition. Mol Cell Biol 33: 874-886, 2013.

6. Mak P, Leav I, Pursell B, Bae D, Yang X, Taglienti CA, Gouvin LM, Sharma VM and Mercurio AM: ERbeta impedes prostate cancer EMT by destabilizing HIF-1alpha and inhibiting VEGF-mediated snail nuclear localization: Implications for Gleason grading. Cancer Cell 17: 319-332, 2010.

7. Cooke VG, LeBleu VS, Keskin D, Khan Z, O'Connell JT, Teng Y, Duncan MB, Xie L, Maeda G, Vong S, et al: Pericyte depletion results in hypoxia-associated epithelial-to-mesenchymal transition and metastasis mediated by met signaling pathway. Cancer Cell 21: 66-81, 2012. 
8. Puisieux A, Brabletz $\mathrm{T}$ and Caramel J: Oncogenic roles of EMT-inducing transcription factors. Nat Cell Biol 16: 488-494, 2014.

9. Ambros V: The functions of animal microRNAs. Nature 431: 350-355, 2004

10. Bartel DP and Bartel DP: MicroRNAs: Genomics, biogenesis, mechanism, and function. Cell 116: 281-297, 2004.

11. Gregory PA, Bert AG, Paterson EL, Barry SC, Tsykin A, Farshid G, Vadas MA, Khew-Goodall Y and Goodall GJ: The miR-200 family and miR-205 regulate epithelial to mesenchymal transition by targeting ZEB1 and SIP1. Nat Cell Biol 10: 593-601, 2008

12. Long H, Wang Z, Chen J, Xiang T, Li Q, Diao X and Zhu B: microRNA-214 promotes epithelial-mesenchymal transition and metastasis in lung adenocarcinoma by targeting the suppressorof-fused protein (Sufu). Oncotarget 6: 38705-38718, 2015.

13. Georges SA, Biery MC, Kim SY, Schelter JM, Guo J, Chang AN, Jackson AL, Carleton MO, Linsley PS, Cleary MA, et al: Coordinated regulation of cell cycle transcripts by p53-inducible microRNAs, miR-192 and miR-215. Cancer Res 68: 10105-10112, 2008.

14. Ansieau S, Morel AP, Hinkal G, Bastid J and Puisieux A: TWISTing an embryonic transcription factor into an oncoprotein. Oncogene 29: 3173-3184, 2010 .

15. Khanbabaei H, Teimoori A and Mohammadi M: The interplay between microRNAs and Twist1 transcription factor: A systematic review. Tumour Biol 37: 7007-7019, 2016.

16. Li X, Xu F, Chang C, Byon J, Papayannopoulou T, Deeg HJ and Marcondes AM: Transcriptional regulation of miR-10a/b by TWIST-1 in myelodysplastic syndromes. Haematologica 98: 414-419, 2013

17. Li X, Zhang Y, Zhang H, Liu X, Gong T, Li M, Sun L, Ji G, Shi Y, Han Z, et al: miRNA-223 promotes gastric cancer invasion and metastasis by targeting tumor suppressor EPB41L3. Mol Cancer Res 9: 824-833, 2011.

18. Lee YB, Bantounas I, Lee DY, Phylactou L, Caldwell MA and Uney JB: Twist-1 regulates the miR-199a/214 cluster during development. Nucleic Acids Res 37: 123-128, 2009.

19. Kajiyama H, Shibata K, Terauchi M, Yamashita M, Ino K, Nawa A and Kikkawa F: Chemoresistance to paclitaxel induces epithelial-mesenchymal transition and enhances metastatic potential for epithelial ovarian carcinoma cells. Int J Oncol 31: 277-283, 2007.
20. Long H, Xie R, Xiang T,Zhao Z, Lin S, Liang Z, Chen Zand Zhu B Autocrine CCL5 signaling promotes invasion and migration of $\mathrm{CD}_{133^{+}}$ovarian cancer stem-like cells via NF- $\mathrm{BB}$-mediated MMP-9 upregulation. Stem Cells 30: 2309-2319, 2012.

21. Schieber MS and Chandel NS: ROS links glucose metabolism to breast cancer stem cell and EMT phenotype. Cancer Cell 23: 265-267, 2013.

22. Qi W, Chen J, Cheng X, Huang J, Xiang T, Li Q, Long H and Zhu B: Targeting the Wnt-regulatory protein CTNNBIP1 by microRNA-214 enhances the stemness and self-renewal of cancer stem-like cells in lung adenocarcinomas. Stem Cells 33: 3423-3436, 2015

23. Reck M, Popat S, Reinmuth N, De Ruysscher D, Kerr KM and Peters S; ESMO Guidelines Working Group: Metastatic non-small-cell lung cancer (NSCLC): ESMO Clinical Practice Guidelines for diagnosis, treatment and follow-up. Ann Oncol 25 (Suppl 3): iii27-iii39, 2014.

24. Chen ZF and Behringer RR: Twist is required in head mesenchyme for cranial neural tube morphogenesis. Genes Dev 9: 686-699, 1995.

25. Mani SA, Guo W, Liao MJ, Eaton EN, Ayyanan A, Zhou AY, Brooks M, Reinhard F, Zhang CC, Shipitsin M, et al: The epithelial-mesenchymal transition generates cells with properties of stem cells. Cell 133: 704-715, 2008

26. Morel AP, Hinkal GW, Thomas C, Fauvet F, Courtois-Cox S, Wierinckx A, Devouassoux-Shisheboran M, Treilleux I, Tissier A, Gras B, et al: EMT inducers catalyze malignant transformation of mammary epithelial cells and drive tumorigenesis towards claudin-low tumors in transgenic mice. PLoS Genet 8: e1002723, 2012.

27. Fan Q, Qiu MT, Zhu Z, Zhou JH, Chen L, Zhou Y, Gu W, Wang LH, Li ZN, Xu Y, et al: Twist induces epithelial-mesenchymal transition in cervical carcinogenesis by regulating the TGF- $\beta /$ Smad3 signaling pathway. Oncol Rep 34: 1787-1794, 2015.

28. Li B, Han Q, Zhu Y, Yu Y, Wang J and Jiang X: Downregulation of miR-214 contributes to intrahepatic cholangiocarcinoma metastasis by targeting twist. FEBS J 279: 2393-2398, 2012.

29. Contreras J and Rao DS: MicroRNAs in inflammation and immune responses. Leukemia 26: 404-413, 2012. 